\title{
ASYMPTOTICALLY STABLE STATIONARY SOLUTIONS TO THE REACTION-DIFFUSION EQUATIONS
}

\author{
ARnold Dikansky
}

We assume that there exists an asymptotically stable stationary solution of a Galerkin approximation for the reaction-diffusion system. It is shown that there exists a nearby stationary solution of the full reaction-diffusion system provided the order of the Galerkin approximation is high enough. The Lyapunov second method is used to prove the asymptotic stability of the stationary solution.

\section{INTRODUCTION}

Systems of reaction-diffusion equations

$$
\frac{\partial u}{\partial t}=D \Delta u+F(u)
$$

with $u \in R^{N}, x \in \Omega \subset R^{n}, D=\operatorname{diag}\left(d_{1}, d_{2}, \ldots, d_{N}\right)$, where each $d_{j}>0$ is a constant, are the main model equations in describing many biological and chemical phenomena. The large time behavior of solutions of reaction-diffusion equations in many aspects resembles one for systems of ordinary differential equations.

Due to the complexity of reaction-diffusion equations their numerical and theoretical studies use Galerkin approximations of these equations. The Galerkin method permits one to associate to the partial differential equation a finite-dimensional system by using the first $m$ eigenfunctions of the operator $D \Delta$ with corresponding boundary conditions. The important question is, of course, to what extent the dynamics of solutions of reaction-diffusion equations as $t \rightarrow \infty$ can be predicted from their Galerkin approximations. In some cases the answer is affirmative if a sufficiently large number of retained eigenfunctions are taken.

For the Navier-Stokes equations Constantin, Foias and Temam [1] showed that, if a solution of a Galerkin equation with a sufficiently large number of retained eigenfunctions of the Stokes operator is almost constant on a time-interval, and some stability spectral condition is satisfied, then there exists a stationary solution of the full NavierStokes equation nearby to the solution of the Galerkin approximation. In their proofs they used spectral properties of linear operators in the Galerkin approximations and the

Received 24th March 1992.

Copyright Clearance Centre, Inc. Serial-fee code: 0004-9729/93 \$A2.00+0.00. 
Navier-Stokes equation linearised about stationary solutions. Kloeden [6] considerably simplified the proofs of some results in [1] using the Lyapunov function defined in a neighbourhood of the stationary solution of the Galerkin equation but he required sufficiently strong asymptotic stability of the stationary solution in order to obtain small perturbations to the Lyapunov function. The Lyapunov second method has been employed to obtain perturbation results for ordinary differential equations by Yoshizawa [8] who developed a lot of relevant machinery. This method was used by Hale [4] for reaction-diffusion equations with the large diffusion and Neumann boundary conditions to show that their solutions are asymptotic to the solutions of an ordinary differential equation which is the Galerkin approximation with the first eigenfunction. Kloeden [7] for the Navier-Stokes equation used the Lyapunov function defined in a neighbourhood of the attractor for the Galerkin equation to establish relations between the existence of the attractor for the Galerkin equation and the attractor of the partial differential equation. For reaction-diffusion equations Dikansky [2] used the Lyapunov functions defined outside of some bounded sets to deduce uniform-boundedness and uniformultimately-boundedness of solutions of the full system from the analogous properties of its Galerkin equation. He also obtained a result similar to one in [1] using spectral properties of linearised operators.

Here for the reaction-diffusion equations we show that if the Galerkin equation with a sufficiently large number of retained eigenfunctions has an asymptotically stable stationary solution $\bar{u}_{m}$, then the reaction-diffusion system has a nearby stationary solution. Using the Lyapunov function defined in a neighbourhood of the stationary solution for the Galerkin equation it is proved that the stationary solution for the reaction-diffusion equation is asymptotically stable. Thus we independently obtain part of the results of [2] to illustrate the broad applicability of the Lyapunov second method to the reaction-diffusion equations. This applicability is especially important when the spectral perturbation methods fail to apply, for example, for more complicated attractors.

In section 2 we outlined necessary results on the Lyapunov second method for stationary solutions as well as reaction-diffusion equations and their Galerkin approximations. Section 3 contains our main result and its proof.

\section{Preliminaries}

A solution of an initial value problem for the ordinary differential equation

$$
\frac{d v}{d t}=f(v), \quad v(0)=v_{0}
$$

will be denoted by $v\left(t ; v_{0}\right)$. 
A stationary solution $\bar{v}$ of $(2.1)(f(\bar{v})=0)$ is (exponentially) asymptotically stable, if there exist constants $\gamma>0$ and $K>0$ and a small neighbourhood $N$ of $\bar{v}$ such that if $v_{0} \in N$, then the solution $v\left(t ; v_{0}\right)$ exists for all $t>0$ and

$$
\left\|v\left(t ; v_{0}\right)-\bar{v}\right\|<K e^{-\gamma t}\left\|v_{0}-\bar{v}\right\| .
$$

Here and elsewhere for $v \in R^{n},\|v\|$ is any norm in $R^{n}$. In order to test the stability of the stationary solution $\bar{v}$ with respect to initial disturbances we introduce the perturbation $w(t) \equiv v(t)-\bar{v}$ into the problem (2.1) and arrive at the following problem for $w(t)$ :

$$
\frac{d w}{d t}=H(w), \quad w(0)=w_{0} .
$$

Here $H(w)=f(\bar{v}+w)-f(\bar{v})$ and $w_{0}=v_{0}-\bar{v}$, so that, in particular, $H(0)=0$ and $w_{0}$ is the initial disturbance away from $\bar{v}$.

The idea of the Lyapunov second method is to detect stability of the zero solution of system (2.3) by means of properties of so called Lyapunov function $V(w)$ and to do this, not directly from a knowledge of the solutions, but indirectly from the sytem (2.3). A Lyapunov function $V(w)$ will be assumed to be a scalar Lipschitz continuous nonnegative function defined in a small neighbourhood $N$ of the asymptotically stable zero solution of the equation (2.3). Corresponding to $V(w)$, we define the derivative $V_{(2.3)}^{\prime}(w)$ of the function $V$ along solutions of equation (2.3):

$$
\begin{aligned}
V_{(2,3)}^{\prime}(w) & \equiv \limsup _{h \rightarrow 0^{+}} \frac{1}{h}[V(w+h H(w))-V(w)] \\
& =\limsup _{h \rightarrow 0^{+}} \frac{1}{h}[V(w(t+h))-V(w(t))] .
\end{aligned}
$$

For the proof of the next result see [8].

Necessary and sufficient conditions for the asymptotic stability of the stationary solution $\bar{w}$ are as follows: There exists a Lyapunov function $V(w)$ which satisfies the following conditions:

$$
\begin{aligned}
& \|w\| \leqslant V(w) \leqslant K\|w\| ; \\
& \left|V(w)-V\left(w^{\prime}\right)\right| \leqslant K\|w\|, \quad w, w^{\prime} \in N \\
& V_{(2.3)}^{\prime}(w) \leqslant-\gamma V(w), \quad \gamma>0 .
\end{aligned}
$$

We note that the constants $K$ and $\gamma$ are the same as in the definition of asymptotic stability of the stationary solution. 
Let $\Omega$ denote an open bounded set of $R^{n}$ with boundary $\Gamma$. For $(x, t) \in \Omega \times R_{+}$ we consider the following reaction-diffusion system involving a vector function $u=$ $\left(u_{1}, u_{2}, \ldots, u_{N}\right)$

$$
\frac{\partial u}{\partial t}=D \Delta u+F(u)
$$

We assume that the nonlinear term $F$ is a $C^{2}$-function. Since we are interested in the long-time behavior of the solutions of (2.7) we modify the nonlinearity in equation (2.7) near $\infty$ assuming that the nonlinear term $F(u)$ satisfies the following condition:

There is $R>0$ such that

$$
F(u)=0 \text { for }\left.|| u\right|_{L^{\infty}} \geqslant R
$$

Let

$$
N_{1}=\sup \{|F(u)|\}, \quad N_{2}=\sup \left\{\left|F^{\prime}(u)\right|\right\}
$$

Equation (2.7) is supplemented with an initial condition

$$
u(x, 0)=u_{0}(x), \quad x \in \Omega,
$$

and a boundary condition of either Dirichlet type or of Neumann type

$$
B[u(x, t)]=0, \quad x \in \Gamma, \quad t \geqslant 0,
$$

where the boundary operator $B$ is either the identity or $\partial u / \partial \nu$. In the case of zero Dirichlet boundary condition we impose a compatibility condition $F(0)=0$.

As usual let $L^{\infty}(\Omega)$ be the space of functions which are defined on $\Omega$ and bounded almost everywhere with norm $|\cdot|_{L^{\infty}}$ and let $H=L_{2}(\Omega)$ be the space of square integrable functions defined on $\Omega$ with the norm ||$_{H}$. We denote by $A$ the linear positive selfadjoint operator on $B$ given by $A u \equiv-\Delta u$ supplemented with boundary conditions (2.11) and having the domain $D(A)=\left\{\phi \in W^{2,2}(\Omega),(2.11)\right.$ holds $\}$. Then $A_{D} \phi \equiv$ $-D A \phi$ is a sectorial operator and one can define the fractional powers $A_{D}^{\alpha}$ of $A_{D}, 0 \leqslant \alpha$ and the space $X^{\alpha}=D\left(A_{D}^{\alpha}\right)$ with the graph norm, ||$_{\alpha}$. If $n \leqslant 3$, and $3 / 4<\alpha<1$, then $X^{\alpha} \subset L^{\infty}(\Omega)$ with continuous inclusion and

$$
|v(\cdot)|_{L^{\infty}} \leqslant k|v(\cdot)|_{X^{\alpha}}, \quad v(x) \in X^{\alpha} .
$$

One can then show (see [4]) that this initial-boundary value problem defines a local $C^{1,1}$ semigroup $S_{t}$ on $X^{\alpha}$ defined by $S_{t} u_{0}=u\left(t, x ; u_{0}\right)$, where $u\left(t, x ; u_{0}\right)$ is a solution of the initial boundary-value problem (2.7), (2.10), (2.11). We shall assume throughout 
the paper that $n \leqslant 3$, and $3 / 4<\alpha<1$. For linear semigroups with the generator $L$ we shall use the exponential notation $e^{L t}$.

It is well known that the operator $A$ is self-adjoint as an operator in $L_{2}(\Omega)$ and the spectrum of $A$ consists of an infinite sequence of eigenvalues $0<\lambda_{1} \leqslant \lambda_{2} \leqslant \ldots$. Because the matrix $D$ is diagonal, each eigenvalue of $A$ corresponds to $N$ eigenvalues of the operator $A_{D}$ therefore there are $N$ eigenvalues $\lambda_{i}^{1}(D), \lambda_{i}^{2}(D), \ldots, \lambda_{i}^{N}(D)$ of the operator $A_{D}$ with corresponding eigenfunctions $\varphi_{i, D}^{1}(x), \varphi_{i, D}^{2}(x), \ldots, \varphi_{i, D}^{N}(x)$, and

$$
\begin{aligned}
& \Lambda_{i, D} \equiv \operatorname{diag}\left(\lambda_{i}^{1}(D), \lambda_{i}^{2}(D), \ldots, \lambda_{i}^{N}(D)\right), \\
& \Lambda_{i, D} \equiv \min \left(\lambda_{i}^{1}(D), \lambda_{i}^{2}(D), \ldots, \lambda_{i}^{N}(D)\right), \quad(i=1,2, \ldots) .
\end{aligned}
$$

We fix an integer $m$ and denote by $P_{m}$ the projection in $L_{2}(\Omega)$ onto the space spanned by the first $m$ eigenvectors of $A_{D}$, and we set $Q_{m}=I-P_{m}$. We recall that $P_{m}$ and $Q_{m}$ commute with $A_{D}$. If $u(x, t)$ is a solution of the initial-boundary value problem (2.7), (2.10), (2.11) we write $p(x, t)=P_{m} u(x, t), q(x, t)=Q_{m} u(x, t)$, so that $u(x, t)=p(x, t)+q(x, t)$. Whenever possible we shall omit the index $m$. Let $X^{\alpha}=P X^{\alpha} \oplus Q X^{\alpha}$. By projection of the equation (2.7) on the invariant subspaces $P X^{\alpha}$ and $Q X^{\alpha}$ we shall find that $p(t)$ and $q(x, t)$ are solutions for $t \geqslant 0$ of the coupled system of equations

$$
\begin{array}{ll}
\frac{d p}{d t}=\Lambda_{D} p+P F(p+q), & p(0)=p_{0} \equiv P u_{0} \\
\frac{\partial q}{\partial t}=D \Delta q+Q F(p+q), & q(x, 0)=q_{0}(x) \equiv Q u_{0}(x) .
\end{array}
$$

Here $p^{k}(x, t)=\sum_{i=1}^{m} p_{i}^{k}(t) \varphi_{i, D}^{k}(x),(i=1,2, \ldots, N)$, where $p_{i}^{k}(t)$ satisfies

$$
\frac{d p_{i}^{k}}{d t}=\Lambda_{k}(D) p_{i}^{k}+\frac{1}{|\Omega|} \int_{\Omega} F^{k}\left(\sum_{i=1}^{m} p_{i}(t) \varphi_{i, D}(x)+q(x, t)\right) \varphi_{i, D}^{k}(x) d x .
$$

Using the variation of constant formula we see that the initial-boundary value problem (2.12), (2.13) is equivalent to the problem

$$
\begin{gathered}
\frac{d p}{d t}=\Lambda_{D} p+P F(p+q), \quad p(0)=p_{0} \\
q(x, t)=e^{L t} q_{0}(x)+\int_{0}^{t} e^{L(t-s)}[Q F(p+q)] d s
\end{gathered}
$$

Here $e^{L t}$ is the linear semigroup corresponding to the problem

$$
\frac{\partial v}{\partial t}=D \Delta v
$$


in $Q X^{\alpha}$ with corresponding boundary conditions. The estimate

$$
\left|e^{L t} u(\cdot)\right|_{\alpha} \leqslant C_{1} t^{-\alpha} e^{-\lambda_{m+1}(D) t}|u(\cdot)|_{H}, \quad t>0,
$$

follows from [5, Theorem 1.5.4]. Using the above notation, the Galerkin approximation of the problem (2.7), (2.10), (2.11) leads to the following system of ordinary differential equations for $u_{m}(t)$

$$
\frac{d u_{m}}{d t}=\Lambda_{D} u_{m}+P F\left(u_{m}\right), \quad u_{m}(0)=p_{0} \equiv P u_{0} .
$$

A stationary solution $\bar{u}_{m}(x)=\left(\bar{u}^{1}(x), \bar{u}^{2}(x), \ldots, \bar{u}^{n}(x)\right)$, where $\bar{u}^{k}(x)=\sum_{i=1}^{m} \bar{u}_{i}^{k}(t) \varphi_{i, D}^{k}(x)$, of the Galerkin system (2.17) satisfies the following equation

$$
\Lambda_{D} \bar{u}_{m}+P F\left(\bar{u}_{m}\right)=0 .
$$

\section{MAIN RESULT.}

THEOREM. For every $\varepsilon>0$ sufficiently small, there exists a natural number $M=$ $M(\varepsilon)$ such that if the Galerkin equation (2.18) for some $m \geqslant M$ has an asymptotically stable stationary solution $\bar{u}_{m}$, then

(a) there exists a stationary solution $\bar{u}(x) \in X^{\alpha}(\Omega)$ of the reaction-diffusion system (2.7) with the boundary conditions (2.11) such that

$$
\left|\bar{u}(\cdot)-\bar{u}_{m}(\cdot)\right|_{X^{\alpha}} \leqslant \varepsilon
$$

(b) the stationary solution $\bar{u}(x)$ is asymptotically stable.

Proof: The proof consists of three parts.

The first part is to show that $p\left(t ; p_{0}\right)$, the $P$-projection of a solution $u\left(x, t ; u_{0}\right)$ of the initial-boundary value problem (2.10), (2.11) for the reaction-diffusion system (2.7) with the initial function $u_{0}(x)$ near $\bar{u}_{m}$, exists for all $t \geqslant 0$ and remains close to $\bar{u}_{m}$, that is,

$$
\left\|\bar{u}_{m}-p\left(t ; p_{0}\right)\right\| \leqslant \delta_{1}
$$

if

$$
\left|\bar{u}_{m}(\cdot)-u_{0}(\cdot)\right|_{X^{\alpha}} \leqslant \varepsilon_{1},
$$

where $\delta_{1}=O\left(\varepsilon_{1}\right)$ with $\varepsilon_{1}$ small enough. 
In order to show that $p\left(t ; p_{0}\right)$ exists for all $t>0$ we introduce the perturbation $p(t)-\bar{u}_{m}$ into problem (2.12), (2.13) and arrive at the following problem for $\left(p(t)-\bar{u}_{m}, q(x, t)\right)$

$$
\begin{aligned}
\frac{d\left(p-\bar{u}_{m}\right)}{d t} & =\Lambda_{D}\left(p-\bar{u}_{m}\right)+P\left[F(p+q)-F\left(\bar{u}_{m}\right)\right] \\
p(0)-\bar{u}_{m} & =p_{0}-\bar{u}_{m}, \\
q(x, t) & =e^{L t} q_{0}(x)+\int_{0}^{t} e^{L(t-s)}[Q F(p+q)] d s .
\end{aligned}
$$

We rewrite the nonlinear term in the right-hand side of (3.3) as follows

$$
\begin{aligned}
& P\left[F(p+q)-F\left(\bar{u}_{m}\right)\right]=P\left[F^{\prime}\left(\bar{u}_{m}\right)\left(p-\bar{u}_{m}\right)\right] \\
& \quad+P\left[F(p+q)-F\left(\bar{u}_{m}\right)-F^{\prime}\left(\bar{u}_{m}\right)\left(p+q-\bar{u}_{m}\right)\right]+P\left[F^{\prime}\left(\bar{u}_{m}\right) q\right] .
\end{aligned}
$$

It follows from (3.2) that if $t$ is small enough then there is a small number $\delta_{2}>0$ such that

$$
\left\|p(t)-\bar{u}_{m}\right\|,|q(\cdot, t)|_{X^{\alpha}}<\delta_{2}
$$

Because $F(u) \in C^{2}$ and due to the representation

$$
g\left(y_{1}\right)-g\left(y_{2}\right)=\int_{0}^{1} g^{\prime}\left(y_{1}+\tau\left(y_{2}-y_{1}\right)\right)\left(y_{2}-y_{1}\right) d \tau
$$

we get

$$
\begin{aligned}
\left\|P\left[F(p+q)-F\left(\bar{u}_{m}\right)-F^{\prime}\left(\bar{u}_{m}\right)\left(p+q-\bar{u}_{m}\right)\right]\right\| & \leqslant o\left(\left|p+q-\bar{u}_{m}\right|_{X^{\alpha}}\right) \\
& \leqslant \eta_{1}\left|p+q-\bar{u}_{m}\right|_{X^{\alpha}}
\end{aligned}
$$

where $\eta_{1}$ is small enough since

$$
\left|p(\cdot, t)+q(\cdot, t)-\bar{u}_{m}(\cdot)\right|_{L^{\infty}} \leqslant|\Omega|^{1 / 2}\left\|p(t)-\bar{u}_{m}\right\|+k|q(\cdot, t)|_{X^{\alpha}} \leqslant \delta_{3}
$$

with $\delta_{3}$ sufficiently small. The last term in the right-hand side of (3.5) is estimated as

$$
\left\|P\left[F^{\prime}\left(\bar{u}_{m}\right) q\right]\right\| \leqslant N_{2}|\Omega|^{1 / 2}|q(\cdot, t)|_{L^{\infty}} \leqslant k N_{2}|\Omega|^{1 / 2}|q(\cdot, t)|_{X^{\alpha}} .
$$

Using (3.7) and the Hölder inequality we get

$$
\begin{aligned}
|Q F(p+q)|_{H} & =|Q[F(p)+F(p+q)-F(p)]|_{H} \\
& \leqslant|F(p)|_{H}+|F(p+q)-F(p)|_{H} \\
& \leqslant N_{1}|\Omega|^{1 / 2}+k N_{2}|\Omega|^{1 / 2}|q(\cdot, t)|_{X^{\alpha}}
\end{aligned}
$$


Defining for $0<\sigma<\lambda_{m+1}(D)$

$$
z(t) \equiv e^{\sigma t}|q(\cdot, t)|_{X \alpha},
$$

from (2.16) and (3.10) we get the following estimate for $z(t)$

$$
\begin{aligned}
z(t) & \leqslant k C_{1} e^{-\left(\lambda_{m+1}(D)-\sigma\right) t} z(0) \\
& +k C_{1} N_{2}|\Omega|^{1 / 2} \int_{0}^{t}(t-s)^{-\alpha} e^{-\left(\lambda_{m+1}(D)-\sigma\right)(t-s)} z(s) d s \\
& +C_{1} N_{1}|\Omega|^{1 / 2} e^{\sigma t} \int_{0}^{t}(t-s)^{-\alpha} e^{-\lambda_{m+1}(D)(t-s)} d s
\end{aligned}
$$

Therefore for

$$
y(t) \equiv \sup _{0 \leqslant s \leqslant t} z(s)
$$

from (3.11) we deduce for $0 \leqslant s \leqslant t$ that

$$
y(t) \leqslant C_{2} e^{-\left(\lambda_{m+1}(D)-\sigma\right) t} z(0)+\zeta_{1} y(t)+\zeta_{2} e^{\sigma t},
$$

where

$$
\begin{aligned}
& K_{1}=\int_{0}^{\infty} s^{-\alpha} e^{-\left(1-\frac{\sigma}{\lambda_{m+1}(D)}\right) \cdot d s}, \quad K_{2}=\int_{0}^{\infty} s^{-\alpha} e^{-\varepsilon} d s, \\
& \zeta_{1}=k K_{1} C_{1} N_{2}|\Omega|^{1 / 2}\left(\lambda_{m+1}(D)\right)^{\alpha-1}, \quad \zeta_{2}=K_{2} C_{1} N_{1}|\Omega|^{1 / 2}\left(\lambda_{m+1}(D)\right)^{\alpha-1} .
\end{aligned}
$$

Inequality (3.12) implies that

$$
y(t)\left(1-\zeta_{1}\right) \leqslant C_{2} z(0)+\zeta_{2} e^{\sigma t}
$$

Therefore

$$
|q(t)|_{X^{\alpha}} \leqslant \frac{C_{2} e^{-\sigma t}|q(0)|_{L^{\infty}}}{1-\zeta_{1}}+\frac{\zeta_{2}}{1-\zeta_{1}}
$$

if $\zeta_{1}<1$. Taking $M$ large enough we shall have $\zeta_{1}<1$, because in the expression defining the constant $\zeta_{1}$ all constants except $\left(\lambda_{m+1}(D)\right)^{\alpha-1}$ do not depend on $m$.

Under the hypothesis that the stationary solution $\bar{u}_{m}$ is asymptotically stable, there exist positive constants $C_{3}$ and $\gamma_{1}$ such that

$$
\left\|e^{A_{m}\left[\bar{u}_{m}\right] t} v\right\| \leqslant C_{3} e^{-\gamma_{1} t}\|v\|, \quad v \in P X^{\alpha},
$$

where $A_{m}\left[\bar{u}_{m}\right] \equiv \Lambda_{D}+P F^{\prime}\left(\bar{u}_{m}\right)$. It easily follows that the constant $C_{3}$ does not depend on $m$. Actually, since the operator $A\left[\bar{u}_{m}\right] \equiv D \Delta+F^{\prime}\left(\bar{u}_{m}\right)$ is a uniformly 
strongly elliptic operator in $\Omega$ then the operator $A\left[\bar{u}_{m}\right]$ with corresponding boundary conditions is a sectorial operator in $X^{\alpha}$ with the norm of its resolvent bounded by a constant $C_{4}$ for large $|\lambda|$ (see [3]). Therefore according to [5, Theorem 1.3.2], the norm of the resolvent of the operator

$$
\Lambda_{D}+P F^{\prime}\left(\bar{u}_{m}\right)=D \Delta+F^{\prime}\left(\bar{u}_{m}\right)-Q\left[D \Delta+F^{\prime}\left(\bar{u}_{m}\right)\right]
$$

is bounded by the constant $C_{3}$ which does not depend on $m, u(\leqslant m)$ is large enough.

As long as (3.6) is satisfied, using the variation of constant formula for (3.3) and estimates (3.5), (3.8), (3.9), (3.15) we have

$$
\begin{aligned}
& \left\|p(t)-\bar{u}_{m}\right\| \leqslant\left\|e^{A_{m}\left[\bar{u}_{m}\right] t}\left(p_{0}-\bar{u}_{m}\right)\right\| \\
& +\left\|\int_{0}^{t} e^{A_{m}\left[\bar{u}_{m}\right] \cdot} P\left[F(p(\cdot, s)+q(\cdot, s))-F\left(\bar{u}_{m}(\cdot)\right)\right] d s\right\| \\
& \leqslant C_{3} e^{-\gamma_{1} t}\left\|p_{0}-\bar{u}_{m}\right\| \\
& +C_{3} \eta_{1} \int_{0}^{t} e^{-\gamma_{1}(t-s)}\left(\|\left[p(s)-\bar{u}_{m} \|+k N_{2} C_{3}|\Omega|^{1 / 2}|q(\cdot, s)|_{X^{\alpha}}\right) d s\right. \\
& \leqslant C_{3} e^{-\gamma_{1} t}\left\|p_{0}-\bar{u}_{m}\right\|+\eta_{2} \sup _{0 \leqslant s \leqslant t}\left\|p(s)-\bar{u}_{m}\right\|+\zeta_{3}<\delta_{1},
\end{aligned}
$$

when $\eta_{2}$ and $\zeta_{3}$ are small enough. If $\left\|p(t)-\bar{u}_{m}\right\|<\delta_{1}$ on $0 \leqslant t<t_{1}$, then either $t_{1}=\infty$ or $\left\|p(t)-\bar{u}_{m}\right\|=\delta_{1}$. But the second case contradicts (3.16), so $p(t)$ exists for all $t>0$ with the estimate $\left\|p(t)-\bar{u}_{m}\right\| \leqslant \delta_{1}$.

The second part of the proof is to show that the full system of reaction-diffusion equations (2.3) has a stationary solution $\bar{u}(x)$ nearby the stationary solution $\bar{u}_{m}$ of the Galerkin equation (2.18) when $M$ is sufficiently large. The stationary solution $\bar{u}(x)$ satisfies the equation

$$
D \Delta \bar{u}+F(\bar{u})=0,
$$

or after projection on the subspaces $P X^{\alpha}$ and $Q X^{\alpha}, \bar{u}(x) \equiv(\bar{p}, \bar{q})$ satisfies the coupled system of stationary equations

$$
\begin{aligned}
\Lambda_{D} \bar{p}+P F(\bar{p}+\bar{q}) & =0 \\
D \Delta \bar{q}+Q F(\bar{p}+\bar{q}) & =0 .
\end{aligned}
$$

Subracting (2.18) from (3.18) we get the system

$$
\begin{aligned}
\Lambda_{D}\left(\bar{p}-\bar{u}_{m}\right)+P F(\bar{p}+\bar{q})-P F\left(\bar{u}_{m}\right) & =0, \\
D \Delta \bar{q}+Q F(\bar{p}+\bar{q}) & =0 .
\end{aligned}
$$


The function $\bar{u}(x)=\bar{p}(x)+\bar{q}(x)$ is a solution of (3.18), (3.19) if and only if $\bar{w}(x) \equiv$ $\bar{u}(x)-\bar{u}_{m}(x)$ is a solution of (3.20), (3.21). So the problem of finding a solution $\bar{u}(x)$ to equation (3.18), (3.19) is equivalent to the one for small solutions $\bar{w}(x)$ to equation (3.20), (3.21). We rewrite system (3.20), (3.21) as follows

$$
\begin{aligned}
\Lambda_{D}\left(\bar{p}-\bar{u}_{m}\right) & +P\left[F^{\prime}\left(\bar{u}_{m}\right)\left(\bar{p}-\bar{u}_{m}\right)\right] \\
& =P\left[F^{\prime}\left(\bar{u}_{m}\right)\left(\bar{p}-\bar{u}_{m}+\bar{q}\right)-F(\bar{p}+\bar{q})+F\left(\bar{u}_{m}\right)\right]-P\left[F^{\prime}\left(\bar{u}_{m}\right) \bar{q}\right],
\end{aligned}
$$

$$
D \Delta \bar{q}+Q F^{\prime}(\widehat{u}) q=Q\left[F^{\prime}(\widehat{u}) \bar{q}-F(\bar{p})\right], \quad \widehat{u}(x) \in X^{\alpha} .
$$

Due to the asymptotic stability of the stationary solution $\bar{u}_{m}$ the linear operator on the left-hand side of (3.22) has an inverse operator from $P_{m} X^{\alpha}$ onto $P_{m} X^{\alpha}$, with its norm bounded by a constant which is independent of $m$ as was explained in the first part of the proof. Using (2.16) and repeating the considerations from the first part of the proof we see that the linear operator in the left-hand side of (3.23) has an inverse operator from $Q X^{\alpha}$ onto itself bounded by $C_{1} /\left(\lambda_{m+1}^{-\alpha+1}-N_{2}\right)$ which is small enough if $M$ is large enough. Therefore system (3.22), (3.23) can be written in the form suitable to apply a fixed-point theorem:

$$
\begin{gathered}
\bar{w}_{m}=F_{1}\left(\bar{w}_{m}, \bar{q}_{m}\right), \\
\bar{q}_{m}=F_{2}\left(\bar{w}_{m}, \bar{q}_{m}\right),
\end{gathered}
$$

with the nonlinear operator $F=\left(F_{1}, F_{2}\right)$ satisfying the conditions of a fixed-point theorem. Thus the fixed point $\bar{u}=\bar{p}+\bar{q}$ is then obtained with $\left\|\bar{p}-\bar{u}_{m}\right\|$ and $|\bar{q}(\cdot)|_{X^{\alpha}}$ small enough provided $M$ is sufficiently large.

The third part of the proof is to show that the stationary solution $\bar{u}(x)$ is asymptotically stable if $M$ is large enough (the definition of asymptotic stability of the stationary solution for the reaction-diffusion equations is analogous to the one for the ordinary differential equations).

The perturbation $v(x, t) \equiv u(x, t)-\bar{u}(x)=p-\bar{p}+q-\bar{q}$ of a solution $u(x, t)$ from the stationary solution $\bar{u}(x)$ satisfies the system

$$
\begin{gathered}
\frac{d(p-\bar{p})}{d t}=\Lambda_{D}(p-\bar{p})+P[F(p+q)-F(\bar{p}+\bar{q})], \\
p(0)-\bar{p} \equiv \widehat{p}_{0}=p_{0}-\bar{p} \\
\begin{aligned}
& \frac{\partial(q-\bar{q})}{\partial t}=D \Delta(q-\bar{q})+Q[F(p+q)-F(\bar{p}+\bar{q})], \\
& q(0)-\bar{q} \equiv \widehat{q}_{0}=q_{0}-\bar{q} .
\end{aligned}
\end{gathered}
$$


We rewrite system (3.26), (3.27) as follows

$$
\begin{aligned}
\frac{d(p-\bar{p})}{d t}= & \Lambda_{D}(p-\bar{p})+P[F(p)-F(\bar{p})] \\
+ & P[F(p+q)-F(\bar{p}+\bar{q})-F(p)+F(\bar{p})], \\
& p(0)-\bar{p}=\widehat{p}_{0}, \\
\frac{\partial(q-\bar{q})}{\partial t}= & D \Delta(q-\bar{q})+Q[F(p+q)-F(\bar{p}+\bar{q})], \\
& q(0)-\bar{q}=\widehat{q}_{0} .
\end{aligned}
$$

From parts 1 and 2 of the the proof and the fact that $|u(\cdot, t)-\bar{u}(\cdot)|_{X^{\alpha}},\left|\bar{u}_{m}(\cdot)-\bar{u}(\cdot)\right|_{X^{\alpha}}$ are small enough, it follows that $p(t)$ and $q(x, t)$ exist for all $t>0$ and $|q(\cdot, t)|_{X^{\alpha}}$ and $|\bar{q}(\cdot)|_{X^{\alpha}}$ are small enough provided $M$ is large enough. Using (3.7) we get

$$
\begin{aligned}
F(p+q) & -F(\bar{p}+\bar{q})-F(p)+F(\bar{p}) \\
& =\int_{0}^{1}\left[F^{\prime}(p+q+\tau(\bar{p}+\bar{q}))(p-\bar{p}+q-\bar{q})-F^{\prime}(p+\tau \bar{p})(p-\bar{p})\right] d \tau \\
& =\int_{0}^{1}\left[F^{\prime}(p+q+\tau(\bar{p}+\bar{q}))-F^{\prime}(p+\tau \bar{p})(p-\bar{p})\right] d \tau \\
& +\int_{0}^{1}\left[F^{\prime}(p+q+\tau(\bar{p}+\bar{q}))(q-\bar{q})\right] d \tau .
\end{aligned}
$$

Because $F \in C^{2}$ the norm of the difference $F^{\prime}(p+q+\tau(\bar{p}+\bar{q}))-F^{\prime}(p+\tau \bar{p})$ is small enough provided $M$ is large enough. Therefore it follows from (3.30) that

$$
\|P[F(p+q)-F(\bar{p}+\bar{q})+F(\bar{p})-F(p)]\| \leqslant \eta_{4}\|p(t)-\bar{p}\|+C_{5}|q(\cdot, t)-\bar{q}(\cdot)|_{\alpha},
$$

where the constant $\eta_{4}$ is small enough provided $M$ is large enough. From (3.26) repeating the considerations from the first part of the proof for the $q$-equation we conclude that

$$
|q(\cdot, t)-\bar{q}(\cdot)|_{X^{\alpha}} \leqslant C_{6} e^{-\gamma_{2} t}\left|q_{0}(\cdot)-\bar{q}(\cdot)\right|_{X^{\alpha}}+\eta_{5}\|p(t)-\bar{p}\|,
$$

where $\gamma_{2}>0$ and the constant $\eta_{5}\left(=O\left(\lambda_{m+1}^{\alpha-1}\right)\right)$ is small enough provided $M$ is large enough.

We shall consider equation (3.28) as a perturbation of the ordinary differential equation (2.3) with $w(t)=p(t)-\bar{p}, H(u)=P F(p)-P F(\bar{p})$. Due to Yoshizawa's theorem there exists the Lyapunov function $V(w)$ which according to (2.5), (3.26), (3.31) satifies the inequality

$$
\begin{aligned}
V_{(3.26)}^{\prime}(p-\bar{p}) & \leqslant V_{(2.18)}^{\prime}(p-\bar{p})+K C_{5} \eta_{5}\|p(t)-\bar{p}\| \\
& +K C_{6} e^{-\gamma_{2} t}\left|q_{0}(\cdot)-\bar{q}(\cdot)\right|_{X^{a}} .
\end{aligned}
$$


Using property (2.6) of the function $V(w)$ we conclude from (3.33) that

$$
V_{(\mathrm{s.26})}^{\prime}(p-\bar{p}) \leqslant-\gamma_{3}\|p-\bar{p}\|+K C_{5} \eta_{5}\|p-\bar{p}\|+K C_{6} e^{-\gamma_{2} t}\left|q_{0}(\cdot)-\bar{q}(\cdot)\right|_{X^{\alpha}},
$$

where $\gamma_{3}>0$. If $K$ does not depend on $m$ with $m \geqslant M$ and $M$ is large enough then we can make

$$
\eta_{5} K C_{5}-\gamma_{3} \leqslant-\gamma_{4}, \quad \gamma_{4}>0
$$

since $\eta_{5}=O\left(\lambda_{m+1}^{\alpha-1}\right)$ which is small provided $M$ is sufficiently large. Therefore

$$
V_{(3.26)}^{\prime}(p-\bar{p}) \leqslant-\gamma_{4}\|p(t)-\bar{p}\|+C_{7} e^{-\gamma_{2} t}\left|q_{0}(\cdot)-\bar{q}(\cdot)\right|_{X \alpha} \cdot
$$

Inequality (3.35) leads to

$$
V(p-\bar{p}) \leqslant C_{8} e^{-\gamma_{5} t}\left|u_{0}(\cdot)-\bar{u}(\cdot)\right|_{X^{\alpha}}, \quad \gamma_{5}>0
$$

Because of property (2.4) for the function $V(w)$ we deduce from (3.36) that

$$
\|p(t)-\bar{p}\| \leqslant C_{9} e^{-\gamma_{5} t}\left|u_{0}(\cdot)-\bar{u}(\cdot)\right|_{X^{\alpha}} \cdot
$$

Finally, combining (3.32) and (3.37) we have

$$
|u(\cdot, t)-\bar{u}(\cdot)|_{X^{\alpha}} \leqslant C e^{-\gamma t}\left|u_{0}(\cdot)-\bar{u}(\cdot)\right|_{X^{\alpha}}, \gamma>0
$$

which proves the asymptotic stability of the stationary solution $\bar{u}(x)$ of the reactiondiffusion system under the assumption that condition (3.34) is satisfied. In the case of the Navier-Stokes equations Kloeden [7] required that it satisfy a condition analogous to condition (3.34) and he called such a stationary solution a sufficiently strongly asymptotic stable solution. Unlike the case of the Navier-Stokes equations, for the reaction-diffusion equations it is easy to show that the constants $K$ and $\gamma_{3}$ do not depend on $m$ if $M$ is large enough, and consequently (3.34) is satisfied. We shall show it using a simple perturbation technique for ordinary differential equations similar to one used in [2] for parabolic equations.

Actually, since the constants $K$ and $\gamma$ in properties (2.4), (2.5), (2.6) of the Lyapunov function are the same as in the definition of asymptotic stability of the stationary solution we shall show that these constants in the definition do not depend on $m$ if $M$ is large enough. We repeat here briefly some considerations from [2].

From the second part of the proof it follows that for each $\ell>m$ there exists a stationary solution $\bar{u}_{l}(x)$ for $l^{\text {th }}$-order Galerkin equation closed to $\bar{u}_{m}$. We consider 
the following equation for perturbations $v_{l}(t)$ around the stationary solution $\bar{u}_{l}(x)$ in $P_{l} X^{\alpha}$

$$
\begin{aligned}
& \frac{d\left(v_{l}-\bar{u}_{l}\right)}{d t}=\Lambda_{D}\left(v_{l}-\bar{u}_{l}\right)+P_{l}\left[F\left(v_{l}\right)-F\left(\bar{u}_{l}\right)\right] \\
& v_{l}(0)-\bar{u}_{l}=P_{l} u_{0}-\bar{u}_{l} .
\end{aligned}
$$

We shall begin with essentially a linear stability analysis of the stationary solution $\bar{u}_{l}$, following by a nonlinear analysis. The coupled system of linearised equations around the stationary solution on the decomposition $P_{l} X^{\alpha}=P_{m} X^{\alpha} \oplus P_{l-m} X^{\alpha}$ is

$$
\begin{aligned}
\frac{d w_{m}(t)}{d t} & =\Lambda_{D} w_{m}+P_{m}\left[F^{\prime}\left(\bar{u}_{l}\right)\left(w_{m}+w_{l-m}\right)\right], \\
w_{m}(0) & =\widehat{w}_{m}, \\
\frac{d w_{l-m}(t)}{d t} & =\Lambda_{D} w_{l-m}+P_{l-m} F^{\prime}\left(\bar{u}_{l}\right)\left(w_{m}+w_{l-m}\right), \\
w_{l-m}(0) & =\widehat{w}_{l-m} .
\end{aligned}
$$

From the assumptions of the theorem (see (3.15)) using Gronwall's inequality it follows that

$$
\left\|\tilde{w}_{m}(t)\right\| \leqslant\left\|e^{\left(\Lambda_{D}+P_{m} F^{\prime}\left[\bar{u}_{l}\right]\right) t} \tilde{w}_{m}(0)\right\| \leqslant C_{10} e^{-\gamma_{0} t}\left\|\tilde{w}_{m}(0)\right\|,
$$

with $\tilde{w}_{m}(t) \in P_{m} X^{\alpha}, \gamma_{\theta}=\gamma_{1} / 2$, when $\left\|\bar{u}_{l}-\bar{u}_{m}\right\|$ is sufficiently small. Hence analogously to the first part of the proof we deduce through the variation of constant formula for $(3.40)$ that

$$
\left\|w_{m}(t)\right\| \leqslant C_{10} e^{-\gamma_{7} t}\left\|w_{m}(0)\right\|+C_{11} \int_{0}^{t} e^{-\gamma_{7}(t-s)}\left\|w_{l-m}(s)\right\| d s
$$

and from (3.41) that

$$
\left\|w_{l-m}(t)\right\| \leqslant C_{12} e^{-\gamma_{8} t}\left\|w_{l-m}(0)\right\|+\eta_{6}\left\|w_{m}(t)\right\|
$$

where $\gamma_{8}>0$ and $\eta_{6}$ is small enough when $M$ is large enough. Substituting the estimate for $\left\|w_{l-m}(t)\right\|$ from (3.43) in the right-hand side of (3.42) and applying methods similar to the ones from the first part of the proof we get the estimate

$$
\left\|w_{m}(t)\right\| \leqslant C_{13} e^{-r_{0} t}\left\|w_{l}(0)\right\|
$$

where $\gamma_{9}>0$. Hence from (3.43), (3.44) we have

$$
\left\|w_{l-m}(t)\right\| \leqslant C_{14} e^{-\gamma_{10} t}\left\|w_{l}(0)\right\|, \quad \gamma_{10}>0
$$


and therefore

$$
\left\|w_{l}(t)\right\| \leqslant C_{15} e^{-\gamma_{11} t}\left\|w_{l}(0)\right\|, \quad \gamma_{11}>0,
$$

where $C_{15}, \gamma_{11}$ do not depend on $m$ if $M$ is large enough.

After having obtained the estimate for the linearised equation the proof of the analogous estimate for the nonlinear ordinary differential equation follows the standard pattern (see [5, Theorem 5.1.1]). Thus

$$
\left\|v_{l}-\bar{u}_{l}\right\| \leqslant C_{16} e^{-\gamma_{12} t}\left\|P_{l} u_{0}-\bar{u}_{l}\right\|
$$

where $C_{16}$ and $\gamma_{12}=\gamma_{11} / 2$ do not depend on $m$ if $M$ is large enough. Because $l$ is any natural number greater than $m$ the constants $K$ and $\gamma$ in the definition of the asymptotic stability of the stationary solution $\bar{u}_{l}$ in (2.2) and consequently in the properties of the Lyapunov function (2.4), (2.5), (2.6) do not depend on $m$.

The theorem has been proved.

\section{REFERENCES}

(1) P. Constantin, C. Foias and R. Temam, 'On the large time Galerkin approximation of the Navier-Stokes equations', SIAM J. Numer. Anal. 21 (1984), 615-634.

[2] A. Dikansky, 'On the large time behavior of the Galerkin approximations to the reaction-diffusion equations', Numer. Funct. Anal. Optim. (submitted).

[3] A.Friedman, Partial differential equations (Holt, Rinehart and Winston, New York, 1969).

[4] J. Hale, 'Large diffusivity and asymptotic behavior in parabolic systems', J. Math. Anal. Appl. 118 (1986), 455-466.

[5] D. Henry, Geometrical theory of semilinear parabolic equations, Lecture Notes in Math. 840 (Springer-Verlag, Berlin, Heidelberg, New York, 1981).

[6] P. E. Kloeden, 'Asymptotically stable attracting sets for the Navier-Stokes equations', Bull. Austral. Math. Soc. 34 (1986), 37-52.

[7] P. E. Kloeden, 'Asymptotically stable solutions of the Navier-Stokes equations and its Galerkin approximations', in Miniconference on nonlinear analysis, (Canberra, 1984), Proc. Centre Math. Anal. Austral. Nat. Univ. (Austral. Nat. Univ., Canberra), pp. 137-150.

[8] T. Yoshizawa, Stability theory by Liapunov's second method (The Math. Soc. of Japan, 1966).

Division of Maths and Computer Science

St. John's University

Staten Island NY 10301

United States of America 